Letter to the Editor

\title{
An Unusual Case of Sinonasal Adenocarcinoma Presenting with a Second Primary in the Larynx
}

\author{
Sandeep Suresh ${ }^{1}$ \\ M. Srinidhi ${ }^{1}$ \\ ${ }^{1}$ Department of Surgical Oncology, Regional Cancer Centre, \\ Thiruvananthapuram, Kerala, India \\ ${ }^{2}$ Department of Pathology, Regional Cancer Centre, \\ Thiruvananthapuram, Kerala, India
}

Elizabeth Mathew lype ${ }^{1}$
Shirish Patil ${ }^{1}$

Rari P. Mony ${ }^{2}$

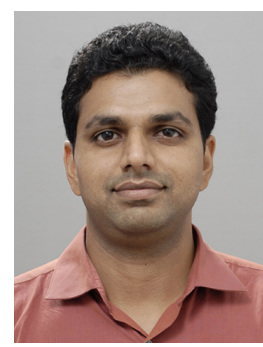

Dr Sandeep Suresh

Address for correspondence M. Srinidhi, MS, Department of Surgical Oncology, Regional Cancer Centre, Thiruvananthapuram, Kerala, India (e-mail: sndp_srsh@yahoo.co.in).

\section{South Asian J Cancer 2020;9:183-185.}

Non-salivary gland type adenocarcinomas of the sinonasal cavity are rare tumors that are often underdiagnosed. They can be broadly divided as the clinically more aggressive intestinal type and less aggressive non-intestinal type. ${ }^{1}$ The more aggressive intestinal variant is associated with a poor prognosis, whereas the low-grade non-intestinal type adenocarcinomas (NIACS) have a good prognosis. Immunohistochemistry plays an important role in distinguishing intestinal from NIACs and also in differentiating them from metastasis from distant sites such as colorectal region.

A 44-year-old male, nonsmoker, without the exposure to wood dust or other chemicals, presented with complaints of blood-tinged nasal discharge from the left nostril, left nasal obstruction, and left-sided headache. A computed tomography scan showed a lesion in the left posterior ethmoid sinuses bulging into the left sphenoid sinus, eroding the cribriform plate with intracranial extradural extension ( - Fig. 1). Biopsy done was in favor of adenocarcinoma. Metastatic workup was negative and no other primary was detected.

Craniofacial resection of the tumor was done using a bicoronal flap incision. Through bifrontal craniotomy and lateral rhinotomy approach, tumor was excised completely with the removal of the cribriform plate. There was no obvious dural invasion or invasion of the lamina papyracea. The final histopathology report was suggestive of low-grade NIAC (-Fig. 2) with immunohistochemistry studies showing CK7 positive and CK20, synaptophysin, chromogranin, thyroid transcription factor-1, and thyroglobulin negative. The patient underwent postoperative radiotherapy (60Gy/30Fractions). The subsequently patient was on regular follow-up, and there was a disease-free interval of 5 years and 2 months.

How to cite this article: Suresh S, Srinidhi M, lype EM, Patil S, Mony RP. An Unusual Case of Sinonasal Adenocarcinoma Presenting with a Second Primary in the Larynx. South Asian J Cancer 2020;9(3):183-185.

DOI https://doi.org/10.1055/s-0040-1721179 ISSN 2278-330X.
The patient then presented to the casualty in acute stridor. Emergency tracheostomy was done. Direct laryngoscopic examination revealed a proliferative mass in the subglottis with fixity of bilateral vocal cords in paramedian position. Biopsy was suggestive of low-grade NIAC. Positron emission tomography-computed tomography scan done showed an abnormal fluorodeoxyglucose uptake of the subglottic lesion; hence, the diagnosis of second primary tumor was made. On imaging lesion was seen in the glottis and subglottis extending to the upper trachea with extralaryngeal spread (-Fig. 3). Total laryngectomy with stapler closure was done. Final histopathology report revealed NIAC with CK7 positive and CK20, synaptophysin, and chromogranin negative on immunohistochemistry studies. The patient is currently on regular follow-up since last one year and is disease-free. Secondary tracheoesophageal prosthesis insertion was done 2 months back for voice rehabilitation.

This case is being reported after obtaining patient consent since such a second primary tumor involving a distinct anatomical site of the head and neck after an interval of 5 years has never been reported in the literature so far.

Adenocarcinomas can be broadly categorized as the clinically more aggressive intestinal type and less aggressive non-intestinal type. The more aggressive intestinal variant is associated with exposure to wood dust and heavy metals like nickel and possibly smoking. Whereas, the low-grade NIACs with a poor prognosis require early diagnosis and treatment. Extensive literature search showed this is the first case of NIAC in two different anatomical sites in the upper aerodigestive tract and probably one among the very few reported cases of NIAC of the larynx. Sinonasal NIACs neither show features of intestinal type nor of salivary gland type of adenocarcinoma.

(C) 2020. Medlntel Services Pvt Ltd.

This is an open access article published by Thieme under the terms of the Creative Commons Attribution-NonDerivative-NonCommercial-License, permitting copying and reproduction so long as the original work is given appropriate credit. Contents may not be used for commercial purposes, or adapted, remixed, transformed or built upon. (https://creativecommons.org/licenses/by-nc-nd/4.0/)

Thieme Medical and Scientific Publishers Pvt. Ltd., A-12, 2nd Floor, Sector 2, Noida-201301 UP, India 


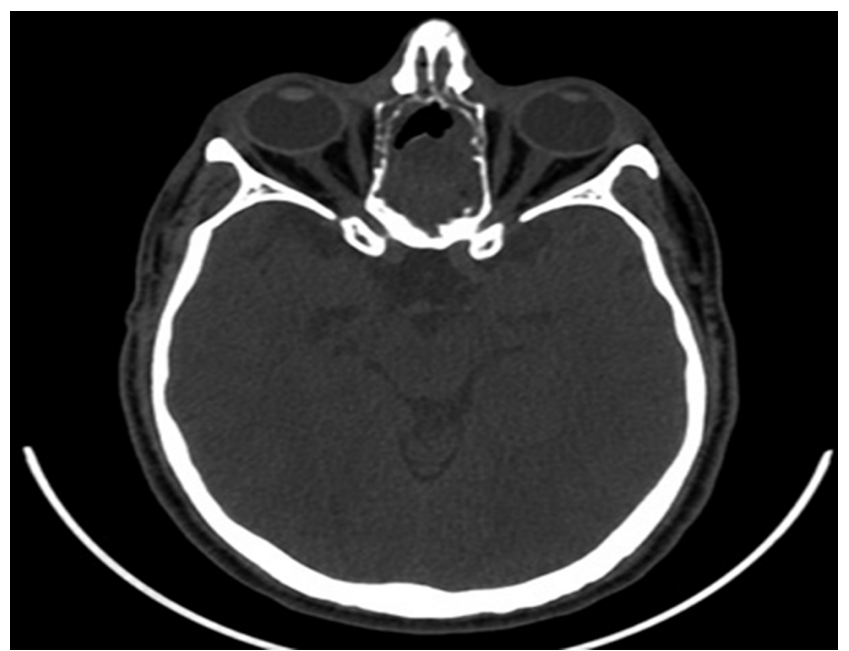

Fig. 1 Computed tomography of sinonasal adenocarcinoma with erosion of the cribriform plate.

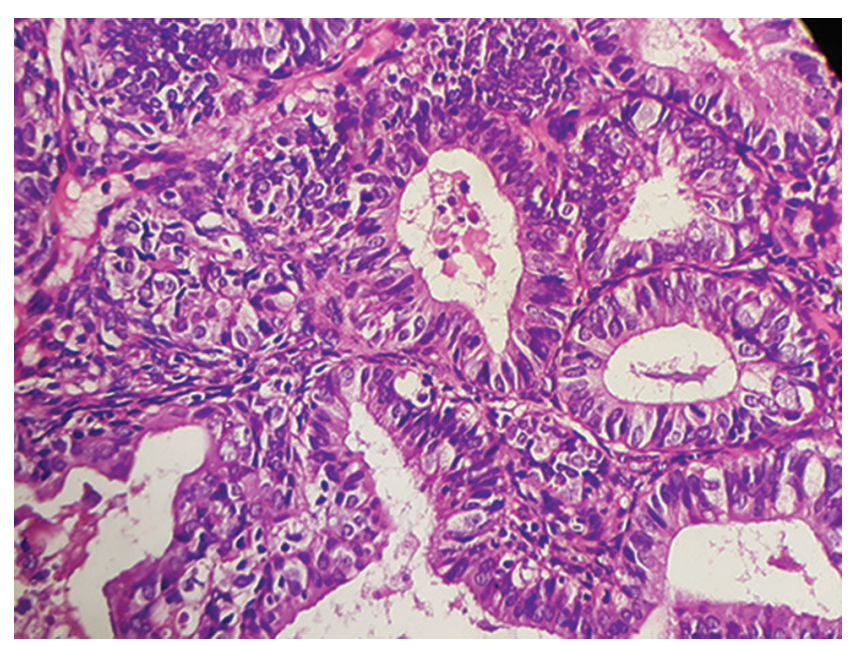

Fig. 2 Microscopic picture of nonintestinal adenocarcinoma showing well-differentiated glandular pattern, compact acini and papillae formation (hematoxylin and eosin, $\mathrm{x} 400$ ).

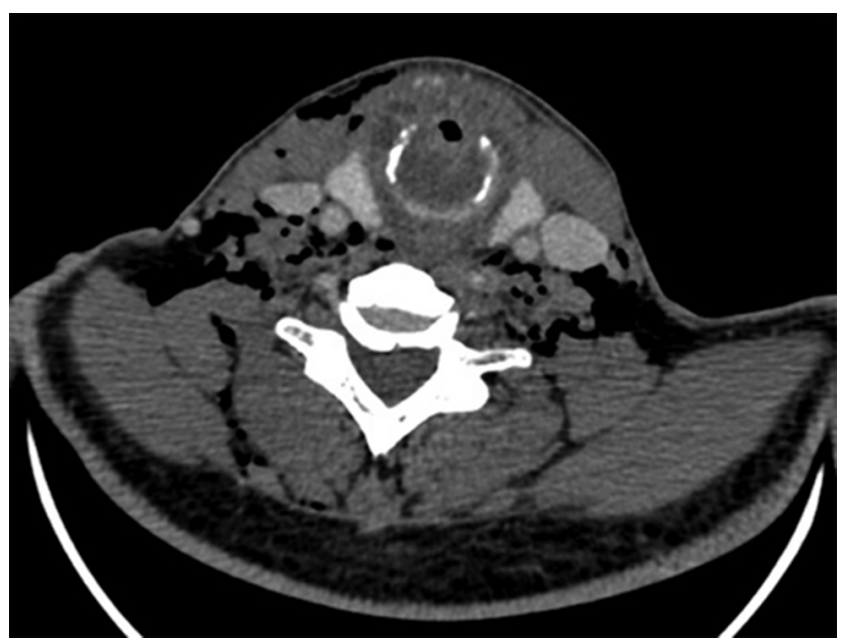

Fig. 3 Computed tomography of adenocarcinoma involving the subglottis causing significant airway compromise.
NIAC usually shows a seromucinous phenotype and is now considered a diagnosis of exclusion. ${ }^{2-4}$ However, there are ongoing studies that aim at early histopathological diagnosis of this clinical entity.

NIAC is further categorized as low grade and high grade. Low-grade NIACs affect individuals 37 to 53 years of age, whereas high-grade NIACs affect individuals more than 60 years of age. Low-grade NIACs constitute only $13 \%$ of sinonasal adenocarcinomas. The most common site affected is the nasal cavity followed by the sinuses (ethmoid sinus more commonly affected than the maxillary sinus). ${ }^{4}$ The most common presentation is nasal obstruction followed by epistaxis. Diagnostic nasal endoscopy may show a polypoidal lesion in the nasal cavity, which may bleed to touch. ${ }^{2}$

Low-grade NIACs exhibit exophytic papillae and tubular or glandular patterns. Their nuclei tend to be pleomorphic and there is mitotic activity on histology. These tumors have a variety of morphologic patterns such as blastomatous, apocrine, oncocytic/mucinous, and poorly differentiated/ undifferentiated. ${ }^{5}$

Differential types of NIAC includes acinic cell carcinoma, salivary-type adenocarcinoma (not otherwise specified), oncocytic Schneiderian NOS papilloma, and, rarely, metastatic papillary carcinoma of the thyroid. Differentiation of NIACs from the intestinal-type of adenocarcinoma on histology is important. On immunohistochemistry, low-grade NIACs are CK7 positive/CK20 negative/CDX-2 negative. However, intestinal-type adenocarcinomas are CK7 positive/CK20 positive/CDX-2 positive, and metastatic colonic adenocarcinomas have CK 7 negative/CK 20 positive/CDX-2 positive immunophenotypes. ${ }^{6}$ In this case, the immunohistochemistry of the laryngeal tumor was in favor of a second primary tumor than metastasis.

Adenocarcinoma of the larynx is uncommon; Whicker et $\mathrm{al}^{7}$ described 12 cases of adenocarcinoma of the larynx that had worst prognosis compared to squamous cell carcinoma and surgery offered best cure. In the retrospective study by Orvidas et al, ${ }^{8} 13$ cases of low-grade NIACs had a disease-free median follow-up of rates 6.5 years following treatment. Similarly in our case report, the disease-free interval before appearance of the second primary was over 5 years. Prognosis depends on the grade of tumor. Sinonasal NIAC without intracranial extension has better prognosis. ${ }^{9}$ Low-grade NIACs have an indolent course with lesser chances of metastasis, whereas high-grade NIACs have greater chances of recurrence and mortality. ${ }^{10,11}$

Surgery remains the mainstay of treatment of sinonasal NIACs. In case of resectable lesions, craniofacial resection offers better chances of survival as compared to conventional surgery. ${ }^{9}$ Extensive disease or high-grade NIACs will require adjuvant radiotherapy. ${ }^{11}$ In this case, radiotherapy was given as an adjuvant treatment following surgery of the sinonasal tumor with intracranial extension. Low-grade NIACs are localized, although local recurrences are possible; however, overall prognosis is favorable. ${ }^{12}$ More than 1 -year survival of our patient following resection of the second primary tumor of the larynx justifies the treatment approach we adopted in this case. 
NIAC of the sinonasal tract following complete treatment after a disease-free interval of more than 5 years, presenting with a second primary tumor in the larynx with similar histology, has never been reported so far. The case reported here is still surviving with a good quality of life following voice rehabilitation.

Low-grade NIACs have a good prognosis. Immunohistochemistry plays a crucial role in distinguishing intestinal from NIACs and also in differentiating them from metastasis of colorectal adenocarcinomas.

Declaration of Patient Consent

The authors certify that they have obtained all appropriate patient consent forms. In the form, the patient(s) has/ have given his/her/their consent for his/her/their images and other clinical information to be reported in the journal. The patients understand that their names and initials will not be published and due efforts will be made to conceal their identity, but anonymity cannot be guaranteed.

Funding

None.

Conflicts of Interest

None declared.

\section{References}

1 Barnes L, Tse LL, Hunt JL, Brandwein-Gensler M, Curtin HD, Boffetta P. World Health Organization Classification of Tumours. Pathology and Genetics Head and Neck Tumours. 3rd edition. Chapter 1: Tumours of Nasal Cavity and Paranasal Sinuses. Lyon: WHO Press; 2005:20-23
2 Bhaijee F, Carron J, Bell D. Low-grade nonintestinal sinonasal adenocarcinoma: a diagnosis of exclusion. Ann Diagn Pathol 2011;15(3):181-184

3 Purgina B, Bastaki JM, Duvvuri U, Seethala RR. A subset of sinonasal non-intestinal type adenocarcinomas are truly seromucinous adenocarcinomas: a morphologic and immunophenotypic assessment and description of a novel pitfall. Head Neck Pathol 2015;9(4):436-446

4 Choi HR, Sturgis EM, Rashid A, et al. Sinonasal adenocarcinoma: evidence for histogenetic divergence of the enteric and nonenteric phenotypes. Hum Pathol 2003;34(11):1101-1107

5 Leivo I. Sinonasal adenocarcinoma: update on classification, immunophenotype and molecular features. Head Neck Pathol 2016;10(1):68-74

6 Song JS, Khang SK, Lee JH, Cho KJ. Sinonasal low-grade adenocarcinoma: report of three cases with the clinicopathologic and immunohistochemical findings. Korean J Pathol 2006;40: 235-240

7 WhickerJH, Neel HB II, Weiland LH, Devine KD. Adenocarcinoma of the larynx. Ann Otol Rhinol Laryngol 1974;83(4):487-490

8 Orvidas LJ, Lewis JE, Weaver AL, Bagniewski SM, Olsen KD. Adenocarcinoma of the nose and paranasal sinuses: a retrospective study of diagnosis, histologic characteristics, and outcomes in 24 patients. Head Neck 2005;27(5):370-375

9 Heffner DK, Hyams VJ, Hauck KW, Lingeman C. Low-grade adenocarcinoma of the nasal cavity and paranasal sinuses. Cancer 1982;50(2):312-322

10 Stelow EB, Mills SE, Jo VY, Carlson DL. Adenocarcinoma of the upper aerodigestive tract. Adv Anat Pathol 2010;17(4):262-269

11 Stelow EB, Jo VY, Mills SE, Carlson DL. A histologic and immunohistochemical study describing the diversity of tumors classified as sinonasal high-grade nonintestinal adenocarcinomas. Am J Surg Pathol 2011;35(7):971-980

12 Cheesman AD, Lund VJ, Howard DJ. Craniofacial resection for tumors of the nasal cavity and paranasal sinuses. Head Neck Surg 1986;8(6):429-435 